\title{
Retinal relaxation following membrane peeling: Effect on vision, central macular thickness, and vector analysis of motion
}

Marc D. de Smet ( $\square$ mddesmet1@mac.com )

University of Amsterdam https://orcid.org/0000-0002-9217-5603

Karina Julian

Cleveland Clinic Abu Dhabi

Jerick Maurin

MIOS

Laurent P. Jolissaint

Haute ecole d'ingenierie et de gestion du canton de Vaud

Marco Mura

King Khaled Eye Specialist Hospital

Research article

Keywords: Epiretinal membrane, ERM, ILM, internal limiting membrane, OCT, Relaxation, SLO, Surgery, Vector analysis

Posted Date: May 22nd, 2019

DOI: https://doi.org/10.21203/rs.2.9163/v2

License: (c) (1) This work is licensed under a Creative Commons Attribution 4.0 International License. Read Full License 


\section{Abstract}

Purpose Removal of an epiretinal membrane (ERM) leads to the outward displacement of retinal vessels and visual improvement. The direction and extent of displacement in the form of a vector analysis over the posterior pole can provide a better understanding of the dynamics and duration of this process, while the magnitude might have implications in terms of visual outcome. Methods Scanning laser ophthalmoscope images of the retina of eyes undergoing ERM peeling were compared before and 6 months after surgery. Stratification was made between prominent and limited displacement, with assessment of visual acuity (VA), and central macular thickness (CMT). In three cases, using the optic nerve as reference, 50 landmarks were chosen within the posterior pole along large and small vascular structures allowing the construction of a vector map of displacement over 1 year. Results Nine eyes with prominent and 6 with limited displacement were assessed. Improvement in VA was similar for both groups, while CMT drop was greatest for the worst group. Vector analysis showed that most vascular movement occurs over the first 6 months, covers most of the posterior retina, is centered around distinct nodes, may lead to several hundred microns of displacement. Conclusions Retinal relaxation has no direct implication on visual recovery. It originates in nodes of retinal contraction. Its extent can be significant

\section{Background}

Epiretinal membranes (ERM) form as a result of fibrocellular proliferation and extracellular matrix deposition at the vitreoretinal junction. These are associated temporally with the development of a posterior vitreous detachment [1]. As the ERM at the surface of the macula contracts, its leads to blurred central vision, aniseikonia, and metamorphopsia [2-5].Contraction at the surface of the retina leads to an increase in central macular thickness (CMT) and is associated with a decrease in best corrected acuity [5].Using fundus images taken at timed intervals, Kofod and la Cour were able to demonstrate, using sectoral grids that tangential retinal vessel movement was correlated with worsening of vision, and that this was not a static but a dynamic process [5].Similar results were observed using autofluorescence imaging [6].

Vitreomacular surgery releases these tractional forces, by eliminating points of attachment between the membrane and the retina and leading to visual improvement over several months [7-9]. On OCT, lowering of CMT is observed, but a return to a completely normal contour with its characteristic foveal depression is seldom realized $[8,10]$.Pre-operative predictive signs of post-operative vision improvement remain difficult to establish. OCT characteristics in patients with ERM have been extensively studied but these have not been found so far to be predictive of visual outcome [11].Based on autofluorescence images before and after vitreoretinal surgery, Nitta et al suggested that if pre-operative hyperfluorescent lines present in a number of ERM eyes disappeared post-operatively, a better visual outcome was possible [12]. The observation was presumed to occur through the relaxation of retinal vessels, assuming their original position. 
A number of studies have attempted to quantify the outward displacement of vessels following surgery using photographic images or scanning laser ophthalmoscopy (SLO) $[5,8,13,14]$. SLO provides a high spatial resolution of the retinal surface with an X-Y precision between 7 and $10 \mu \mathrm{m}$. If rotational aberrations are taken into account, it is possible to superimpose images taken at different time points. By comparing pre-and post-operative images taken in eyes undergoing macular peeling procedures for ERM, it is evident that retinal displacement varies greatly between eyes. Some vessels exhibit significant displacement while in other eyes, a limited shift is observed. This process is not only evident in perifoveal vessels, on close observation, it is also present in vessels located close to the vascular arcade. A commonly used technique in engineering and physical sciences is the use of vectors to indicate intensity and direction of a process of interest. We decided to apply this technique to vascular relaxation following macular pucker surgery. . The aim of this study was to better understand the vascular relaxation process and to see if it correlated in any way with improvement in vision. We attempted to correlate the large or limited vascular shifts observed in patients with visual outcomes, and used vector matrices to quantify at multiple retinal vascular landmarks (50 per eye) the direction and extent of vascular displacement over time. This approach confirmed that vascular displacement occurs over a large area. It defined a time course for this displacement extending over months, and as might be expected, displacement is greatest around the various nodes of fibrosis on the retinal surface.

\section{Methodology}

An electronic database was searched for patients with a diagnosis of epiretinal membrane, having undergone an epiretinal membrane peel, with 6 or more months of follow-up in whom spectral domain SLO-OCT images were available for analysis. Patients with prior ocular surgeries except in cases of cataract removal, or with additional macular pathologies were excluded from further analysis. Informed consent was obtained on all patients when they first presented to the clinic with regards to the future analysis of data collected, for the purpose of biomedical research, provided it was anonymized, according to a procedure and form that was approved by the local ethics commission. As this was a retrospective study, no further approval was required from the local ethics commission.

At each visit, as part of routine patient follow-up, a complete ocular exam was obtained including the measurement of best corrected visual acuity using a wall mounted Snellen chart, and the recording of a spectral domain SLO-OCT topography map centered on the fovea using a series D SLO-OCT Opko device (Tampa Florida, USA). For the purpose of the current analysis, the following data was recorded: sex, age at surgery, state of the lens, vision (pre-operative, post-operative at 1, 3, 6 months, and when possible at 1 year), central macular thickness (CMT) taken from the topographical map centered on the fovea at each of the above mentioned time points.

Patients were operated by the same surgeon (MdS) under local anesthesia. Following a 25G pars plana vitrectomy, the epiretinal membrane was peeled over an area extending up to both temporal arcades. The surface of the retina was stained with either membrane or brilliant blue to confirm the removal all membranes including the ILM. 
Patients were stratified based on the degree of vascular displacement observed when the pre-operative SLO image taken from the topography map was superimposed on the image obtained 6 months following surgery. A significant shift was considered to have taken place if any of the vessels surrounding the fovea, including the vessels at the arcade had a displacement of more than $150 \mu \mathrm{m}$ (the diameter of the temporal vein $2 \mathrm{~mm}$ proximal to its insertion into the optic nerve) $[15,16]$. Other patients were considered to have minimal change.

SLO images of three representative patients where further analyzed to determine the magnitude and direction of the retinal shift over time. A patient with a minimal shift and two patients with a significant retinal shift were selected. In one patient with a significant shift, there was a significant decrease in CMT while in the other there was not. For each time point, an SLO image centered on the fovea was chosen from the corresponding B-scan file in which there was minimal visible artifacts in scan lines, or obscuration of the image. Snapshot images of the appropriate scan were exported as tiff files.

A full description of the analysis procedure is provided in the supplemental digital content

file A. Images were first analyzed to exclude SLO images in which horizontal shearing had taken place during data acquisition. The images were normalized by their root mean square value after adjustment for shadows generate by floaters in the vitreous or from the tear film. Images were superimposed on one another using the center of the optic nerve as a reference point. The edge of the optic nerve was used to compensate for translational and rotational shifts as needed. Displacement of individual junctional points along vessels were used to build a vector distortion map across the retina. The coordinates of these junctions were determined manually (LPJ). On each image, about 50 reference points were selected, distributed between broad to thin vessels from both the arterial and venous systems. Vectors units were transformed from pixels to millimeters on the retina using a scaling factor measured on the images, assuming a $0.15 \mathrm{~mm}$ for a large venule near the optic nerve [17].

Statistical analysis was carried out using Prism (GraphPad Software, La Jolla, CA) version 5.0d for MacOS. A non parametric Mann-Whitney test was used to compare groups.

\section{Results}

A total of 15 eyes of 15 patients (9 males and 6 females), average age of 74 years (SD \pm 7 years) met the inclusion criteria. Nine eyes had evidence of significant vascular displacement while 6 eyes showed little displacement. Vision improvement was noted in both groups of patients during the initial 6 post-operative months (figure 1). Patients with prominent vascular displacement started with a lower average acuity (mean $0.4[20 / 50] \pm 0.3$ ) compared to the group experiencing less displacement (mean $0.6[20 / 30] \pm 0.3$ ), but this difference was not statistically significant. The rate of improvement in vision was comparable between the two groups over 6 months. In both groups, improved vision on average by two to three lines. Improvement was more consistent in the patients experiencing less vascular displacement. 
Initial central macular thickness was highest in the eyes experiencing prominent vascular displacement $424 \pm 171 \mu \mathrm{m}$ (median $424 \mu \mathrm{m}$ ) versus $382 \pm 94 \mu \mathrm{m}$ (median $400 \mu \mathrm{m}$ ), but this difference was not statistically significant. While central macular thickness decreased in most eyes, in only one eye did it return to normal values (figure 2). In none of the eyes, did the foveal contour return to normal. In eyes with prominent vascular displacement, the largest drop in central macular thickness occurred in 4 of 8 eyes within the first month, and was present by 3 months in $7 / 8$ eyes. Four eyes had an increase in thickness due to the presence of macular edema one month after surgery, as evidenced by the presence of cystoid spaces on the macular OCT image. This edema when present took several weeks to resolve. In many cases, the fovea remained thickened even after complete resolution of the foveal fluid. In eyes with minimal displacement, macular edema was observed in only 1 of 6 eyes. While most of the reduction in CMT started shortly after surgery, the major drop in CMT started in some eyes as late as month 3.

The vectors corresponding to the direction and degree of retinal relaxation are shown for the three selected cases in figure 3 . Vascular landmarks moved centrifugally away from the points of contraction. In case $A$, there were at least 2 sites of contraction but little outward displacement in the area of the fovea. On OCT, the traction was located around the fovea causing the formation of a pseudolamellar hole (figure 4). This patient's vision improved to 0.9 [20/25++] from 0.6 [20/30-]. In case B, at least 3 foci of relaxation are seen ( 2 temporal to the fovea and 1 inferonasal). On OCT, the fovea is thickened and is covered by a membrane. However, most of the outward displacement occurs at a distance from the foveal center. Outward displacement of vessels is noted at the arcade and beyond. Vision improved to 0.8 [20/25] from 0.6 [20/30-]. While CMT decreased following surgery, most of the loss in height occurred temporal to the fovea and inferiorly, in the area corresponding to the vascular shift. In case $\mathrm{C}$, the superficial contraction is located adjacent to the temporal edge of the fovea and is centered in a single focus. The outward shift extends to the arcade, and is mainly located on the temporal side of the fovea. Vision improved from 0.6 [20/30-] to 1.0 [20/20] (figure 4).

The cumulated average shift over time is depicted in figure 3 . The shift is greatest in the initial few months but continues for the length of follow-up in all three patients. In cases B and C, measurements were also obtained at 1 year post surgery. Further relaxation was observed in patient $B$ but was not observed in patient $\mathrm{C}$, though the magnitude of this further relaxation was significantly lower than in the initial 6 months.

\section{Discussion}

Peeling epiretinal membranes leads to a release of centripetal tractional forces and allows the retina to regain a more normal position. Several attempts have been made to quantify such movements using retinal landmarks based on retinal photography, SLO imaging, or fundus autofluorescence $[5,6,8,13,14]$. Using large vascular landmarks, it is possible to measure the general outward motion of the retina, comparing the size of pre and post operative surface areas bound by major vessels or landmarks [6]. Outward displacement of arterioles and venules from the foveal center or changes in the length of vessels between two branching points can also be determined and quantified per quadrant [14]. Major 
vascular shifts occurred in a temporal rather than nasal direction, as might be expected given the physical limitation posed by the optic nerve. However, in some cases such as macular hole closures following the removal of an epiretinal membrane and tamponade with gas, a nasal shift of the foveal center has be observed on OCT. Such shifts, averaging $150 \mu \mathrm{m}$, were not observed with spontaneous closures, and was also not observed in the current study [18].Scan laser ophthalmoscopes generate images with a high spatial resolution in the order of $10 \mu \mathrm{m}$ in the XY plane [19]. Therefore, it is possible to analyze in each patient, images taken at different time points both qualitatively and quantitatively, provided that imaging artifacts caused by shearing, rotation, tilting or magnification are eliminated. By averaging and normalizing 20 images taken on the same day, most aberrations can be eliminated. The use the optic nerve as a common reference point allows for the alignment of images taken on different days. Using the same reference area, it is possible to eliminate any rotational artifact. Having corrected for tilt, rotation and size, it is then possible to use vectors to determine the direction and degree of vascular shift over an extended area of the retinal surface. When plotted over time, the rate of change can be determined.

Extensive puckers were associated with a lower initial vision, and a lower visual outcome, though the degree of vision recovery over a 6 month period was comparable to patients with less extensive epiretinal membranes. Limitations to vision recovery were related in a number of cases to the presence of macular edema, often absent in the first month after surgery, but appearing subsequently, as previously described [20].This was also the origin of observed increases in retinal thickness, or the absence of a drop in thickness following surgery. It was observed equally in patients with prominent or limited vascular displacement following the removal of a membrane. Macular edema following membrane peeling has been reported in 1 to $10 \%$ of cases depending on the series [21]. Two distinct mechanisms are proposed. The first, as in Irvine Gass syndrome is related to the release of inflammatory mediators and is possibly related to the use of peripheral laser along the vitreous base [21]. Characteristically, it presents with outer retinal cystoid spaces. The second mechanism is related to pre-existing or surgically induced damage to the Muller cells and is characterized by intraretinal cyst formation not associated with fluorescein leakage, or with leakage visible in the very late stage. Both mechanisms, but predominantly the second were observed in our patients.

The detailed analysis of vascular shifts at the retinal surface in three patients revealed that following surgery, relaxation takes place over an extended period of time. However, the bulk of the shift has occurred by 6 months. A study published in 2016, showed that the time course of improvement in metamorphosia following ERM peeling in patients followed over a 2 year period was most intense in the initial 6 months [22]. We observed that relaxation emanated from the points of fibrosis on the retinal surface and lead to a localized lowering in retinal thickness. The rate of relaxation appears to be variable. Additional cases would be necessary to determine whether this is dependent on the severity and extent of the superficial retinal distortions present prior to surgery. There appears to be limited or no correlation between retinal relaxation and improvement in vision. Indeed all three patients had the same improvement in vision though the variation in vascular shift was considerable. Vascular tortuosity and 
post operative vascular shifts are reflective of changes at the retinal surface, while vision is more clearly related to changes in the ellipsoid zone and at the external limiting membrane [23-26].

Given our observations, one may question the need for extensive peels over the whole macular surface. Relieving traction at specific nodes of retinal fibrosis might eliminate traction and its deleterious effects on deeper retinal structures while avoiding the risk of damaging the nerve fiber layer [27]. Indeed a metaanalysis carried out in 2017, showed little difference in visual outcome with and without ILM peeling [28]. ILM peeling was associated with a lesser rate of recurrence, though an increase central macular thickness was more frequently observed following an ILM peel.

As with all observational studies, its major limitation is the number of patients studied. In order to gain more insight on the characteristics of retinal relaxation following an epiretinal peel, it would be necessary to study a larger number of patients. Given the time involved in obtaining the measurements carried out in this study, automation of image acquisition and the determination of the vascular shifts at bifurcation points of retinal vessels would be particularly useful. With the advent of OCT angiography, it might also be of interest to study vascular shifts in peri-foveal capillary beds, particularly at the level of the deeper capillary plexus as it may be reflective of the tractional forces exerted on the photoreceptor layer. Further studies may elucidate these questions.

\section{Declarations}

\section{Ethics approval and consent to participate:}

This was a retrospective data analysis. For this analysis no informed consent was required.

\section{Availability of data and material:}

All data used in the current study will be made available in an appropriate database repository upon acceptance of the article.

Competing interest: None with regards to this work.

MD de Smet is a consultant for Alcon, Allergan, Abbvie, Horama, NightStaRx, Oxular, Sanofi, Santen, Tarsisus Medical; he has received lecture fees from Allergan, Sanofi, Santen in the alst 2 years; he is a copatent holder with Thrombogenics and Preceyes; he has received research funding in the last 2 years from Alcon, Allergan, Preceyes.

M Mura: is a consultant for Alcon and has received both research funding and lecture fees from the same company.

\section{Funding: None}

Authors' contributions: Marc D. de Smet was responsible for the design of the study, data review and writing of the manuscript. Karina Julian was responsible for the selection of cases and analysis of the 
OCT and visual results. Jerick Maurin performed all data collection on patients as well as vision measurements. LP Jolissaint did the vector analysis and selected the cases best suited for the analysis from the provided dataset. M Mura helped with the analysis, writing, and critical review of the manuscript.

Acknowledgements: None

\section{Bibliography}

1. Snead DR, James S, Snead MP. Pathological changes in the vitreoretinal junction 1: epiretinal membrane formation. Eye 2008;22:1310-1317.

2. Suh MH, Seo JM, Park KH, Yu HG. Associations between macular findings by optical coherence tomography and visual outcomes after epiretinal membrane removal. Am J Ophthalmol. 2009;147:473480 .

3. Koo HC, Rhim WI, Lee EK. Morphologic and functional association of retinal layers beneath the epiretinal membrane with spectral-domain optical coherence tomography in eyes without photoreceptor abnormality. Graefes Arch Clin Exp Ophthalmol. 2012;250:491-498.

4. Okamoto F, Sugiura Y, Okamoto Y, Hiraoka T, Oshika T. Time Course of Changes in Aniseikonia and Foveal Microstructure after Vitrectomy for Epiretinal Membrane. Ophthalmol. 2014;121:2255-2260

5. Kofod M, la Cour M Quantification of retinal tangential movement in epiretinal membranes. Ophthalmol. 2012;119:1886-1891.

6. Rodrigues IA, Lee EJ, Williamson TH. Measurement of retinal displacement and metamorphopsia after epiretinal membrane or macular hole surgery. Retina. 2016; 36:695-702.

7. Dogramaci M, Williamson TH. Dynamics of epiretinal membrane removal off the retinal surface: a computer simulation project. Br J Ophthalmol. 2013;97:1202-1207.

8. Yang HK, Kim SJ, Jung YS, Kim KG, Kim JH, Yu HG. Improvement of horizontal macular contraction after surgical removal of epiretinal membranes. Eye. 2011;25:754-761.

9. Yang HS, Kim JT, Joe SG, Lee JY, Yoon YH. Postoperative restoration of foveal inner retinal configuration in patients with epiretinal membrane and abnormally thick inner retina. Retina. 2015;35:111-119.

10. Kim JH, Kang SW, Kong MG, Ha HS. Assessment of retinal layers and visual rehabilitation after epiretinal membrane removal. Graefes Arch Clin Exp Ophthalmol. 2013;251:1055-1064 .

11. Scheerlinck LM, van der Valk R, van Leeuwen R. Predictive factors for postoperative visual acuity in idiopathic epiretinal membrane: a systematic review. Acta Ophthalmol. 2015;93: 203-212. 
12. Nitta E, Shiraga F, Shiragami C, Fukuda K, Yamashita A, Fujiwara A. Displacement of the Retina and Its Recovery After Vitrectomy in Idiopathic Epiretinal Membrane. Am J Ophthalmol. 2013;155: 1014-1020.

13. Weinberger D, Stiebel-Kalish H, Priel E, Barash D, Axer-Siegel R, Yassur Y. Digital red-free photography for the evaluation of retinal blood vessel displacement in epiretinal membrane. Ophthalmol. 1999;106:1380-1383

14. Kim JW, Choi KS. Quantitative analysis of macular contraction in idiopathic epiretinal membrane. BMC Ophthalmol. 2014;14:51.

15. Garcia-Ortiz L, Recio-Rodriguez JI, Parra-Sanchez J, Gonzalez Elena LJ, Patino-Alonso MC, AgudoConde $\mathrm{C}$, Rodriguez-Sanchez E, et al. A new tool to assess retinal vessel caliber. Reliability and validity of measures and their relationship with cardiovascular risk. J Hypertens. 2012;30:770-777 .

16. Wang $\mathrm{S}, \mathrm{Xu} \mathrm{L}$, Wang $\mathrm{Y}$, Wang $\mathrm{Y}$, Jonas JB. Retinal vessel diameter in normal and glaucomatous eyes: the Beijing eye study. Clin Exp Ophthalmol. 2007;35:800-807.

17. Knudtson MD, Klein BEK, Klein R, Wong TY, Hubbard LD, Lee KE, Meuer SM, Bulla CP. Variation associated with measurement of retinal vessel diameters at different points in the pulse cycle. $\mathrm{Br} J$ Ophthalmol. 2004;88:57-61.

18. Kawano K, Ito Y, Kondo M, Ishikawa K, Kachi S, Ueno S, Iguchi Y, Terasaki H. Displacement of foveal area toward optic disc after macular hole surgery with internal limiting membrane peeling. Eye. 2013;27:871-877.

19. van Velthoven MEJ, Verbraak FD, Yannuzzi LA, Rosen RB, Podoleanu AG, de Smet MD (2006) Imaging the retina by en face optical coherence tomography. Retina 26:129-136

20. Sigler EJ, Randolph JC, Charles S. Delayed onset inner nuclear layer cystic changes following internal limiting membrane removal for epimacular membrane. Graefes Arch Clin Exp Ophthalmol. 2013;251:1679-1685.

21. Frisina R, Pinackatt SJ, Sartore M, Monfardini A, Baldi A, Cesana BM, Semeraro F, Bratu A, Parolini B. Cystoid macular edema after pars plana vitrectomy for idiopathic epiretinal membrane. Graefes Arch Clin Exp Ophthalmol. 2015;253:47-56.

22. Kinoshita T, Imaizumi H, Miyamoto H, Katome T, Semba K, Mitamura Y. Two-year results of metamorphopsia, visual acuity, and optical coherence tomographic parameters after epiretinal membrane surgery. Graefes Arch Clin Exp Ophthalmol. 2016;254:1041-1049.

23. de Smet Marc D. de Smet. Insights into the physiopathology of inflammatory macular edema. Dev Ophthalmol. 2017;58:168-177. 
24. Ito $\mathrm{S}$, Miyamoto $\mathrm{N}$, Ishida $\mathrm{K}$, Kurimoto $\mathrm{Y}$. Association between external limiting membrane status and visual acuity in diabetic macular oedema. Br J Ophthalmol. 2013;97:228-232.

25. Phadikar P, Saxena S, Ruia S, Lai TY, Meyer CH, Eliott D. The potential of spectral domain optical coherence tomography imaging based retinal biomarkers. Int J Retina Vitreous. 2017;3:1.

26. Inoue M, Morita S, Watanabe Y, Kaneko T, Yamane S, Kobayashi S, Arakawa A, Kadonosono K. Inner segment/outer segment junction assessed by spectral-domain optical coherence tomography in patients with idiopathic epiretinal membrane. Am J Ophthalmol. 2010;150:834-839.

27. Tadayoni R, Paques M, Massin P, Mouki-Benani S, Mikol J, Gaudric A. Dissociated optic nerve fiber layer appearance of the fundus after idiopathic epiretinal membrane removal. Ophthalmol. 2001;108:2279-2283.

28. Fang $X L$, Tong $Y$, Zhou $Y L$, Zhao $P Q$, Wang ZY. Internal limiting membrane peeling or not: a systematic review and meta-analysis of idiopathic macular pucker surgery. Br J Ophthalmol. 2017;101:1535-1541.

\section{Figures}




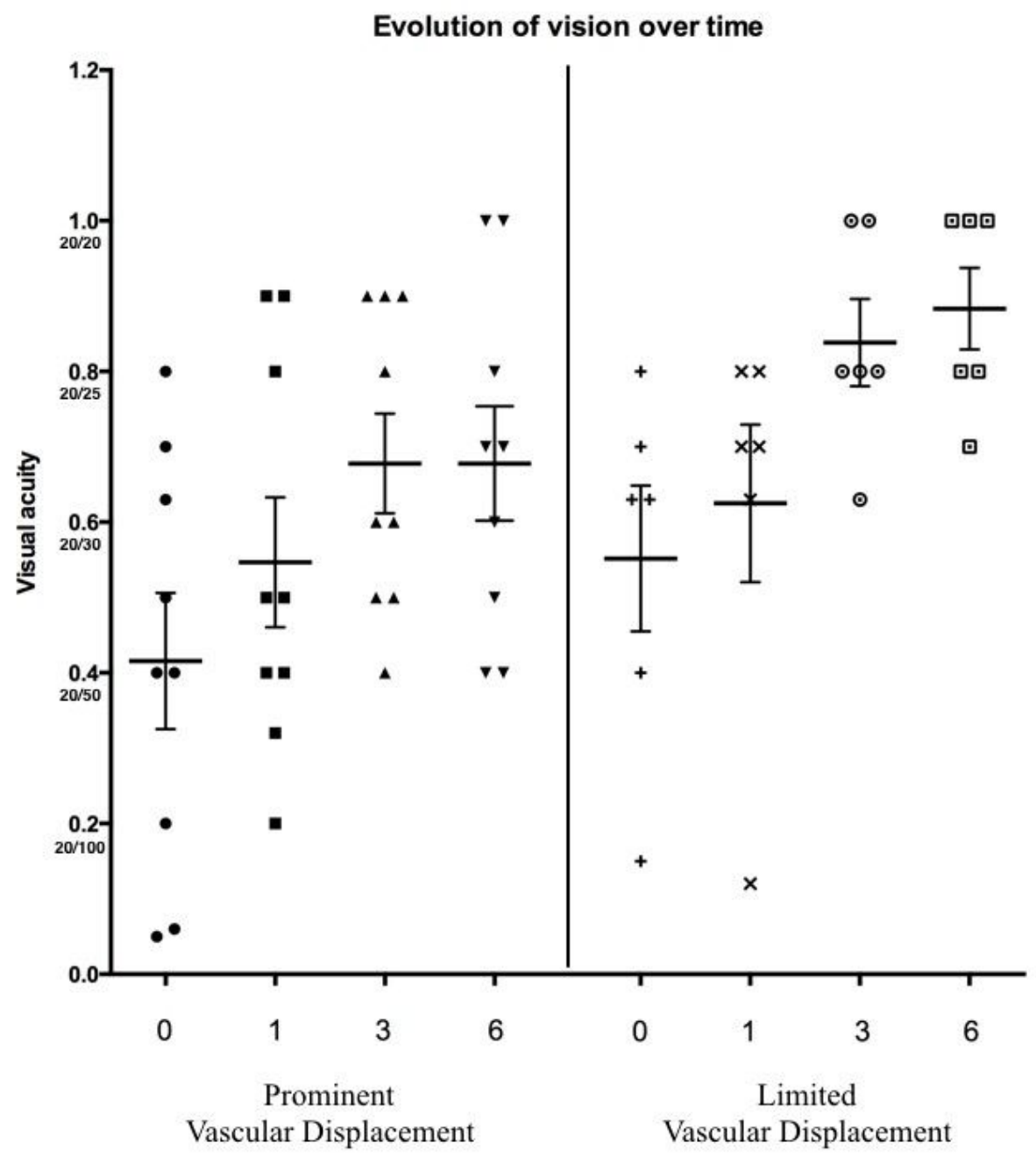

Time (months) since the surgical intervention

Figure 1

Evolution of vision over time. (Equivalent Snellen notation given under the decimal value) 


\section{Evolution of central macular thickness}



Figure 2

Evolution of the central macular thickness $(\mu \mathrm{m})$ over time. The letters correspond to the patients in whom vector analysis was carried out in figure 3. 

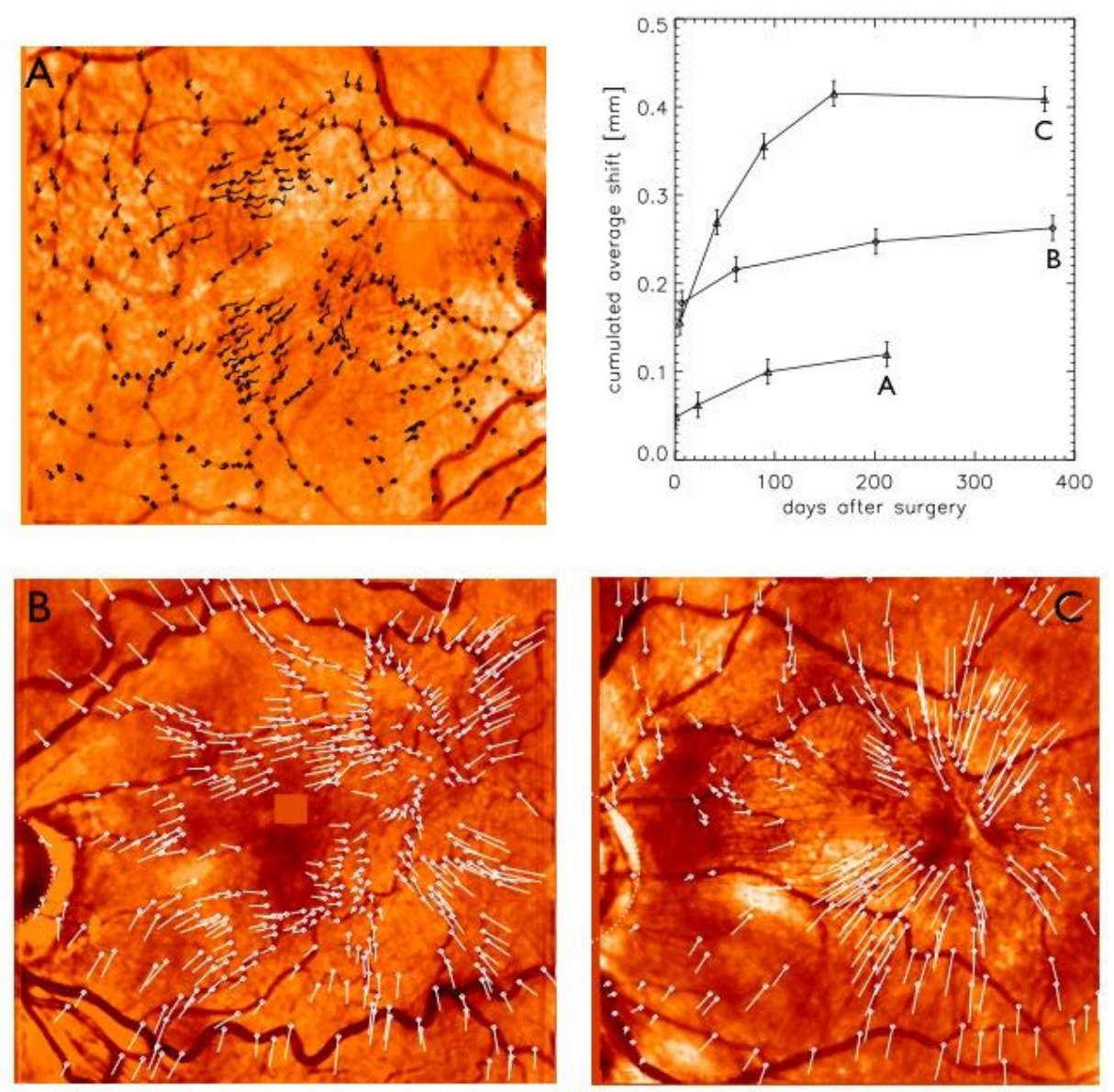

\section{Figure 3}

Vector representation of surface vascular movement in select patients and graphical representation of cumulated average shifts over the analyzed time period per patient. (For each patient, the final image post-operative image was compared to the pre-operative SLO image to generate the vector representations. Each line represents the displacement and direction of representative points). 

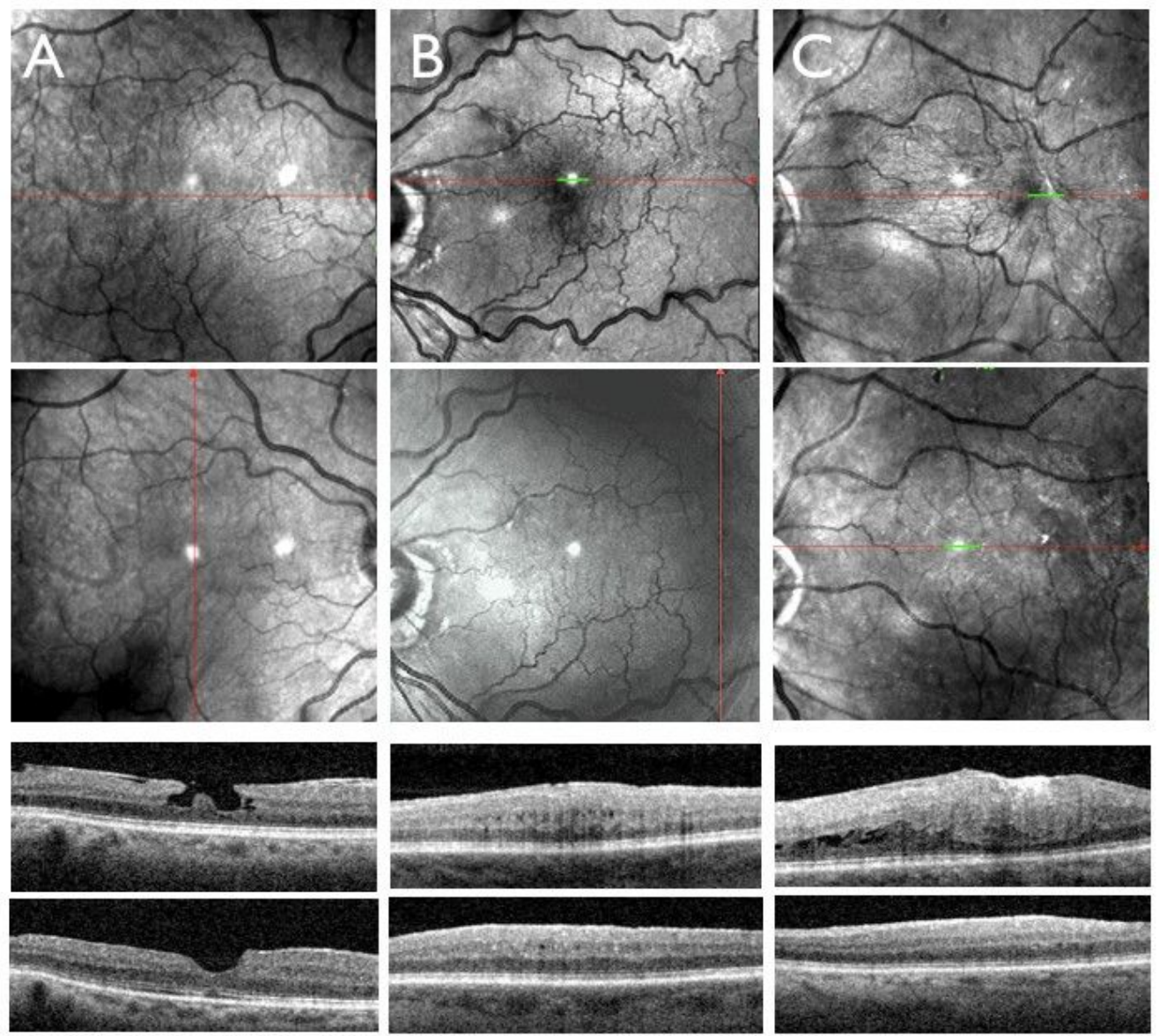

Figure 4

SLO and central OCT scans take of patients studied in figure 3. The upper SLO panels show the preoperative scan, the lower SLO panels the last post-operative scan. In the OCT scan, the upper panel corresponds to a central macular scan prior to surgery, while the lower panel corresponds to the scan taken at the end of the study.

\section{Supplementary Files}

This is a list of supplementary files associated with this preprint. Click to download.

- supplement1.docx 